\title{
ROTURA DE LA UNIÓN MUSCULO TENDINOSA DISTAL DEL BÍCEPS BRAQUIAL Distal Brachial Biceps Musculotendinous Rupture
}

\section{*Roberto Pineda Leiva, **Oscar Benítez, ***Dalton Argean Norwood, ****Annie Isabel Navarro}

\section{RESUMEN}

Las roturas de la unión músculo-tendinosa son frecuentes en deportista, aunque en concreto, la del músculo bíceps braquial distal es muy rara. El objetivo de este trabajo es presentar un caso clínico de rotura músculo-tendinosa del bíceps braquial distal y el tratamiento aplicado. Presentamos el caso clinico de paciente masculino de 17 años de edad que mientras jugaba futbol su miembro superior izquierdo quedo atrapado en una verja y al tratar de liberarlo con movimientos bruscos, experimentó dolor de inicio súbito en el tercio distal de su brazo, de carácter punzante, intensidad 10/10 en la escala nominal del dolor, sin atenuantes, exacerbándose con la pronación, supinación y flexión del miembro superior. El mecanismo del trauma coincide con una elongación de su articulación glenohumeral con el codo extendido y el antebrazo supinado. El paciente fue manejado conservadoramente y con ejercicios de rehabilitación ambulatorios. A los tres meses de la rotura obtuvo una puntuación de 100 con

*Cirujano Plástico, Profesor Titular de la Escuela Universitaria de Ciencias de la Salud de la Universidad Nacional Autónoma de Honduras (EUCS-UNAHVS), Jefe del Departamento de Ciencias Básicas.

**Ortopeda y Traumatólogo Hospital Centro Médico SA (CEMESA) y Hospital Militar

***Estudiante V año, Carrera de Medicina y Cirugía, EUCS, UNAH-VS.

****Medico General, egresada de la EUCS, UNAH-VS.

Correspondencia a daltonnorwood2@hotmail.com rpl3030@yahoo.com el Mayo Elbow Performance Score (MEPS), demostrando que en estos casos está altamente indicado el tratamiento conservador con buenos resultados funcionales.

\section{PALABRAS CLAVE}

Codo, Sistema Musculoesqueletico, Traumatismo de los Tendones.

\section{ABSTRACT}

The breakage of muscle-tendon junction are common in athletes, but in particular the distal biceps brachial muscle is very rare. The aim of this work is to present a case of rupture of the muscle-tendon distal biceps brachial, the treatment applied. We present the case of male patient 17 years of age while playing football his left upper limb was trapped in a fence and trying to free it with jerky movements, experienced sudden onset pain in the distal third of the arm, throbbing character, $10 / 10$ at the nominal intensity scale unmitigated pain, exacerbated with pronation, supination and flexion of the upper limb. The mechanism of trauma coincides with an extension of his glenohumeral joint with the elbow extended and the forearm supinated.

The patient was managed conservatively and outpatient rehabilitation exercises. Three months break I got a score of 100 
with the Mayo Elbow Performance Score (MEPS). Proving that in these cases it is strongly indicated conservative treatment with good functional results.

\section{KEYWORDS}

Elbow, Musculoskeletal System, Tendon Injuries.

\section{INTRODUCCIÓN}

Las roturas de la unión músculo-tendinosa son frecuentes en deportistas ${ }^{(1-4)}$ aunque la del músculo bíceps braquial distal es rara y existen escasas referencias en la literatura. Por el contrario, la avulsión del bíceps distal de la tuberosidad radial es una lesión frecuente ${ }^{(5-9)}$ que puede ser confundida con una rotura músculo-tendinosa. ${ }^{(4)}$ El objetivo de este trabajo es presentar un caso clínico de rotura músculo-tendinosa del bíceps braquial distal y el tratamiento aplicado.

\section{CASO CLINICO}

Paciente de sexo masculino, de 17 años de edad, sin antecedentes patológicos previos, con historia de que mientras jugaba futbol fue empujado contra un portón, quedando su extremidad izquierda atrapada en las verjas hasta el tercio medio de su brazo experimentando dolor intenso en su codo, que no cedió con analgésicos ni al inmovilizarlo pasivamente.

En la exploración física presentó dolor a la movilización y palpación anterior y distal del codo, sin asociar pérdida de fuerza distal ni alteraciones sensitivas, los pulsos distales radial, ulnar y mediano se encontraron conservados. Ver figura $\mathrm{N}^{\circ} 1$.

\section{Figura $\mathrm{N}^{\circ} 1$ :}

Lesión del miembro superior izquierdo.

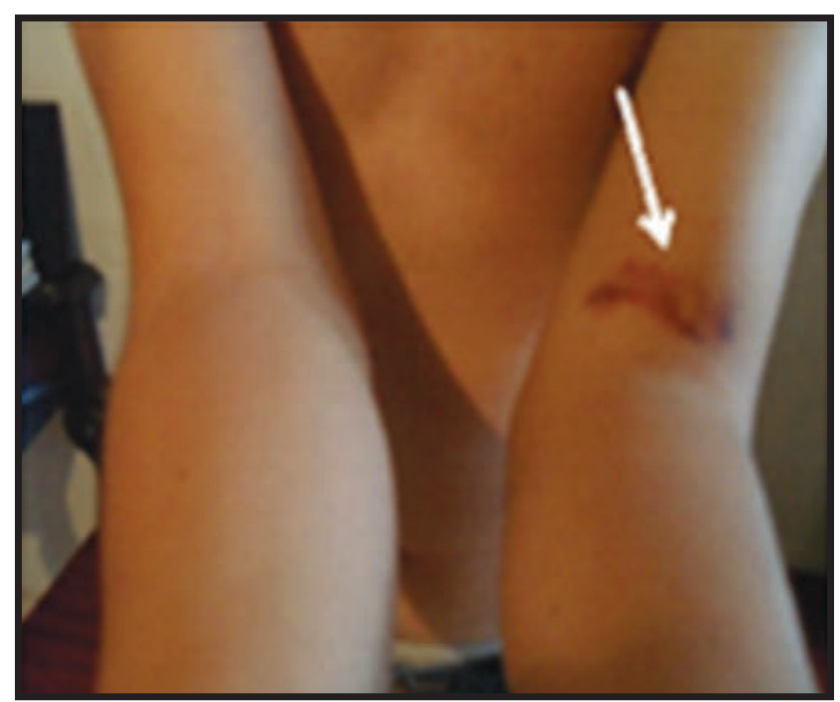

Se observa lesión en miembro superior izquierdo, con área de equimosis y cambios inflamatorios.

Se realizó radiografía descartando fractura, estudio ecográfico e imagen por resonancia magnética (IRM) que constató la rotura del músculo bíceps en su unión miotendinosa distal. Ver Figura $\mathrm{N}^{\circ} 2$.

Figura $N^{\circ}$ 2:

IRM del miembro superior del paciente

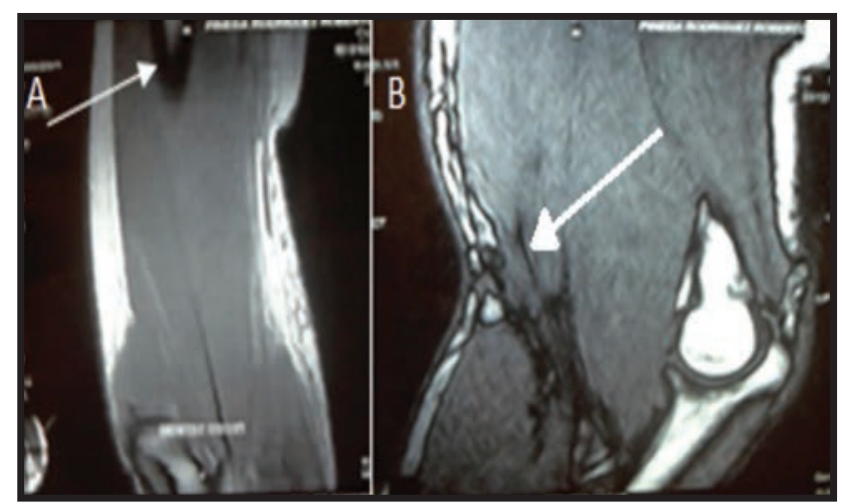

Se observa: A). El punto de la lesionen la unión musculo tendinosa del bíceps braquial.

B). Vista lateral de la rotura de la unión musculo tendinosa del bíceps.

Se inmovilizó la extremidad con cabestrillo y se inició tratamiento con analgésico oral, 
permitiendo movilizaciones activas asistidas cada día del codo. Se alternaron aplicaciones de compresas frías y tibias al momento de sus ejercicios.

Se retiró el cabestrillo a las 3 semanas, permitiendo movilizaciones activas. La rehabilitación se realizo ambulatoriamente y el paciente se reincorporó a sus actividades laborales y deportivas. Se le recomendó controles.

A los tres meses, presentaba flexo-extensión y prono-supinación completa y a pesar de referir una ligera disminución de fuerza respecto a su situación previa a la lesión, no encontró dificultades para realizar las actividades de su vida diaria y deportiva, con puntuación de 100 puntos sobre 100 de acuerdo con el Mayo Elbow Performance Score (MEPS).

\section{DISCUSION}

Las lesiones del tendón distal del bíceps, son poco frecuentes y se trata de una avulsión de la inserción del tendón en la tuberosidad bicipital de radio. Si bien el diagnóstico y tratamiento de esta última entidad ha sido ampliamente estudiado, la rotura de la unión músculo-tendinosa esta escasamente descrita en la literatura.

La única serie que hemos encontrado corresponde a Schamblin y Safran con 6 casos. ${ }^{(4)}$ Según Morrey, se trata de una lesión que tiene pre dilección por personas con encefalopa-tía. ${ }^{(5)}$ Esta observación no se ha descrito en ninguno de los casos de la serie de Schamblin y Safran quienes, sin embargo, describen que en tres de los seis casos existía un antecedente de levantamiento de pesos de forma recreacional. En nuestro caso tampoco encontramos antecedente de encefalopatía y sí un mecanismo de elongación gleno-humeral con extensión de codo y supinación de antebrazo.

Schamblin y Safran presentaron resultados buenos y excelentes a los tres años de seguimiento sin realizar tratamiento quirúrgico (5 casos puntuaron 100 y uno puntuó 85 en MEPS). ${ }^{(4)}$ Los autores remarcan que no se trata de «no hacer nada» sino que el paciente debe realizar una fisioterapia dirigida para obtener una recuperación funcional satisfactoria.

El paciente que estamos presentando, fue manejado conservadoramente y con ejercicios de rehabilitación ambulatorios. A los tres meses de la rotura obtuvo una puntuación de 100 con el Mayo Elbow Performance Score, (MEPS). ${ }^{(9)}$ Demostrando que en estos casos está altamente indicado el tratamiento conservador con buenos resultados funcionales.

En conclusión, podemos esperar un buen resultado funcional en las roturas de la unión miotendinosa distal del bíceps sin realizar tratamiento quirúrgico. Sin embargo, son necesarios más estudios para determinar el tratamiento de elección. 


\section{BIBLIOGRAFÍA}

1. Garrett WE Jr. Muscle strain injuries. Am J Sport Med. 1996; 24(6Suppl): 2-8.

2. Palmer WE, Kuong SJ, Elmadbouh HM. MR imaging of myotendinous stain. ARJ Am J Roentgent. 1999; 173:703-73.

3. Schamblin ML, Safran MR. Injury of the dis- tal biceps at the musculotendinous juncti- on. Shoulder Elbow Surg 2007; 16:208-12.

4. Cohen S, Bradley J. Acute proximal hamstring rupture. J Am Acad Orthop Surg 2007; 15:350-5.

5. Morrey BF. The elbow and its disorders. Philadelphia: Saunders; 2000.
6. De Smet AA, Best TM. MR imaging of the distribution and location of acute hamstring injuries in athletes. Am J Roentgenol 2000; 174:393-9.

7. Klonz A, Loitz D, Reilmann H. Proximal and distal ruptures of the biceps brachii tendon. Unfallchirurg 2003; 106:755-63.

8. Alanis LM, Zamora PM, Cruz A. Ruptura distal del tendón del bíceps. Reporte de un caso. Acta Ortop Mex 2009; 23:228-31.

9. López-Zabala I, Muñoz-Mahamud E, Ballesteros J, Fernández-Valencia JA. Distal biceps musculotendinous rupture. Report of two cases. Trauma Fund MAPFRE. 2011;223:164-167. 